\title{
Proof of the (local) angular momentum-mass inequality for axisymmetric black holes
}

\author{
Sergio Dain \\ Max-Planck-Institut für Gravitationsphysik, Am Mühlenberg 1, 14476 Golm, Germany
}

Received 30 June 2006

Published 20 October 2006

Online at stacks.iop.org/CQG/23/6845

\begin{abstract}
We prove that for any vacuum, maximal, asymptotically flat, axisymmetric initial data for Einstein equations close to extreme Kerr data, the inequality $\sqrt{J} \leqslant m$ is satisfied, where $m$ and $J$ are the total mass and angular momentum of the data. The proof consists in showing that extreme Kerr is a local minimum of the mass.
\end{abstract}

PACS numbers: 04.70.Bw, 04.20.Dw, 04.20.Ex, 04.20.Fy

\section{Introduction}

Physical arguments led to the conjecture that for every axisymmetric, vacuum, asymptotically flat, complete, initial data set for Einstein equations the following inequality should hold:

$$
\sqrt{|J|} \leqslant m
$$

where $m$ is the mass of the data and $J$ is the angular momentum in the asymptotic region. Moreover, the equality in (1) should imply that the data are the slice of the extreme Kerr black hole. For a more detailed discussion of the motivations and relevance of (1) and related inequalities see [5-8, 10].

In [6], the proof of (1), for maximal data, was reduced to a variational problem. In this paper, we will prove that this variational problem has a local minimum at the extreme Kerr solution, and hence we prove that inequality (1) is satisfied for axisymmetric, maximal, vacuum data sufficiently close to extreme Kerr data.

We first present the mathematical formulation of the variational problem. Let $\rho, z, \phi$ be cylindrical coordinates in $\mathbb{R}^{3}$, and let $v, Y$ be the two functions which depend only on $\rho, z$. Consider the functional defined in $[6]^{1}$

$$
\mathcal{M}(v, Y)=\frac{1}{32 \pi} \int_{\mathbb{R}^{3}}\left(|\partial v|^{2}+\rho^{-4} \mathrm{e}^{-2 v}|\partial Y|^{2}\right) \mathrm{d} \mu,
$$

where $\mathrm{d} \mu=\rho \mathrm{d} z \mathrm{~d} \rho \mathrm{d} \phi$ is the volume element in $\mathbb{R}^{3}$ and $\partial$ denotes the partial derivative with respect to $\rho$ and $z$, that is $|\partial v|^{2}=v_{, z}^{2}+v_{, \rho}^{2}$. Let $v_{0}$ and $Y_{0}$ denote the extreme Kerr initial

1 We have slightly changed the notation in [6]: we rescale $v \rightarrow 4 v$. 
data given explicitly in appendix A. These functions depend on a free parameter $J$, the angular momentum of the data. Set

$$
v=v_{0}+\alpha, \quad Y=Y_{0}+y .
$$

To simplify the notation, we will write $\varphi \equiv(\alpha, y), u \equiv(v, Y)$ and $u_{0} \equiv\left(v_{0}, Y_{0}\right)$. Let $\Omega$ be a (unbounded) domain in $\mathbb{R}^{3}$. We introduce the following weighted spaces of $C^{1}$ functions in $\Omega$ :

$$
\|f\|_{C_{\beta}^{1}(\Omega)}=\sup _{x \in \Omega}\left\{\sigma^{-\beta}|f|+\sigma^{-\beta+1}|\partial f|\right\},
$$

with $\beta<-1 / 2$ and $\sigma=\sqrt{r^{2}+1}, r=\sqrt{\rho^{2}+z^{2}}$.

Let $\rho_{0}>0$ be a constant and $K_{\rho_{0}}$ be the cylinder $\rho \leqslant \rho_{0}$ in $\mathbb{R}^{3}$. We define the domain $\Omega_{\rho_{0}}$ by $\Omega_{\rho_{0}}=\mathbb{R}^{3} \backslash K_{\rho_{0}}$. The perturbation $y$ is assumed to vanish in $K_{\rho_{0}}$. This is consistent with the physical requirement that the perturbations keep fixed the angular momentum $J$ (see equation (17) and also $[6,5])$. The Banach space $\mathcal{B}$ is defined by

$$
\|\varphi\|_{\mathcal{B}} \equiv\|\alpha\|_{C_{\beta}^{1}\left(\mathbb{R}^{3}\right)}+\|y\|_{C_{\beta}^{1}\left(\Omega_{\rho_{0}}\right)} .
$$

We consider $\mathcal{M}$ as a functional on $\mathcal{M}: \mathcal{B} \rightarrow \mathbb{R}$. The following is the main result of this paper.

Theorem 1.1. The functional $\mathcal{M}: \mathcal{B} \rightarrow \mathbb{R}$ defined by (2) has a strict local minimum at $u_{0}$. That is, there exist $\epsilon>0$ such that

$$
\left.\mathcal{M}\left(u_{0}+\varphi\right)\right\rangle \mathcal{M}\left(u_{0}\right)
$$

for all $\varphi \in \mathcal{B}$ with $\|\varphi\|_{\mathcal{B}}<\epsilon$ and $\varphi \neq 0$.

For simplicity, we have assumed that $y$ vanished at $K_{\rho_{0}}$; the theorem is expected to be true if we impose appropriate decay conditions for $y$ at the axis; however, the analysis in this case is more complicated. We also expect that $u_{0}$ is an absolute global minimum of $\mathcal{M}$ in $\mathcal{B}$ (see [6]).

We discuss now the application of theorem 1.1 to the proof of inequality (1) in a neighbourhood of extreme Kerr data. What follows is a summary of the results presented in [6]. A maximal initial data set for Einstein's vacuum equations consists in a Riemannian metric $\tilde{h}_{a b}$ and a trace-free symmetric tensor field $\tilde{K}^{a b}$ such that the vacuum constraint equations

$$
\begin{aligned}
& \tilde{D}_{b} \tilde{K}^{a b}=0, \\
& \tilde{R}-\tilde{K}_{a b} \tilde{K}^{a b}=0,
\end{aligned}
$$

are satisfied, where $\tilde{D}_{a}$ and $\tilde{R}$ are the Levi-Civita connection and the Ricci scalar associated with $\tilde{h}_{a b}$. In these equations, the indices are moved with the metric $\tilde{h}_{a b}$ and its inverse $\tilde{h}^{a b}$.

We will assume that the initial data are axially symmetric, that is, there exists an axial Killing vector $\eta^{a}$ such that

$$
\mathfrak{£}_{\eta} \tilde{h}_{a b}=0, \quad \mathfrak{£}_{\eta} \tilde{K}_{a b}=0,
$$

where $£$ denotes the Lie derivative.

The Killing vector $\eta^{a}$ is assumed to be hypersurface orthogonal. Under these conditions, the metric $\tilde{h}_{a b}$ can be characterized by two functions $q, v$ of the coordinates $\rho, z$. These functions are specified as follows. We write the metric in the form

$$
\tilde{h}_{a b}=\mathrm{e}^{v} h_{a b}
$$


where the conformal metric $h_{a b}$ is given by

$$
h=\mathrm{e}^{-2 q}\left(\mathrm{~d} \rho^{2}+\mathrm{d} z^{2}\right)+\rho^{2} \mathrm{~d} \phi^{2} .
$$

In these coordinates, we have $\eta^{a}=(\partial / \partial \varphi)^{a}$ and its norm with respect to the metric $\tilde{h}_{a b}$ will be denoted by $X$ :

$$
X=\eta^{a} \eta^{b} \tilde{h}_{a b}=\mathrm{e}^{v} \rho^{2} .
$$

The function $q$ is assumed to be smooth with respect to the coordinates $\rho, z$. At the axis, we impose the regularity condition

$$
q(\rho=0, z)=0,
$$

and at infinity, we assume the following fall-off:

$$
q=o\left(r^{-1}\right), \quad q_{, r}=o\left(r^{-2}\right) .
$$
$\tilde{S}^{a}$ by

The potential $Y$ is calculated in terms of the second fundamental form. Define the vector

$$
\tilde{S}_{a}=\tilde{K}_{a b} \eta^{b}-X^{-1} \tilde{\eta}_{a} \tilde{K}_{b c} \eta^{b} \eta^{c}
$$

where $\tilde{\eta}_{a}=\tilde{h}_{a b} \eta^{b}$. Using equations (7), (9) and the Killing equation we obtain

$$
\tilde{D}_{[b} K_{a]}=0, \quad K_{a}=\tilde{\epsilon}_{a b c} \tilde{S}^{b} \eta^{c},
$$

where $\tilde{\epsilon}_{a b c}$ is the volume element of $\tilde{h}_{a b}$. Then there exists a scalar function $Y$ such that $K_{a}=\tilde{D}_{a} Y$. This function contains the angular momentum $J$ of the data

$$
J=\frac{1}{8}(Y(\rho=0,-z)-Y(\rho=0, z)), \quad z \neq 0 .
$$

For any data $\left(\tilde{h}_{a b}, \tilde{K}^{a b}\right)$ which satisfy these assumptions, we have a pair $v, Y$ and its corresponding angular momentum $J$. Let $u_{0} \equiv\left(v_{0}, Y_{0}\right)$ be the extreme Kerr data with the same $J$ and define $\varphi \equiv(\alpha, y)$ by (3). We have the following result.

Corollary 1.2. Let $\left(\tilde{h}_{a b}, \tilde{K}^{a b}\right)$ be a maximal, axisymmetric, vacuum, initial data with mass $m$ and angular momentum $J$, such that the metric satisfies (10)-(11) and (13)-(14). Define $\varphi$ as above. Then, there exists $\epsilon>0$ such that for $\|\varphi\|_{\mathcal{B}}<\epsilon$ the inequality (1) holds. Moreover, $m=\sqrt{J}$ in this neighbourhood if and only if the data are the extreme Kerr data.

Proof. In [6], it has been proved that under the present assumptions we have

$$
m \geqslant \mathcal{M}\left(u_{0}+\varphi\right) \text {. }
$$

By theorem 1.1, we have

$$
\left.m \geqslant \mathcal{M}\left(u_{0}+\varphi\right)\right\rangle \mathcal{M}\left(u_{0}\right),
$$

for $\varphi \neq 0$. Since $u_{0}$ is the extreme Kerr data, we have $\mathcal{M}\left(u_{0}\right)=\sqrt{|J|}$ (see [6]), then the conclusion follows.

Finally, we mention that these results are similar in spirit to the local proof of the positivity of the mass given in [2, 4]. 


\section{Preliminaries}

Let us assume $\varphi \in \mathcal{B}$ and consider the real-valued function

$$
i_{\varphi}(t)=\mathcal{M}\left(u_{0}+t \varphi\right) .
$$

The first derivative of $i_{\varphi}(t)$ with respect to $t$ is given by

$$
i_{\varphi}^{\prime}(t)=\frac{1}{16 \pi} \int_{\mathbb{R}^{3}}\left\{\partial v \partial \alpha+\left(\partial Y \partial y-\alpha|\partial Y|^{2}\right) X^{-2}\right\} \mathrm{d} \mu,
$$

where the $t$ dependence on the right-hand side of (21) is encoded in the functions $v, Y, X$ defined by

$$
v \equiv v(t)=v_{0}+t \alpha, \quad Y \equiv Y(t)=Y_{0}+t y
$$

and

$$
X \equiv X(t)=\rho^{2} \mathrm{e}^{v(t)}, \quad X_{0} \equiv X(0)=\rho^{2} \mathrm{e}^{v_{0}} .
$$

The second derivative is given by

$$
i_{\varphi}^{\prime \prime}(t)=\frac{1}{16 \pi} \int_{\mathbb{R}^{3}}\left\{|\partial \alpha|^{2}+\left(2 \alpha^{2}|\partial Y|^{2}-4 \alpha \partial Y \partial y+|\partial y|^{2}\right) X^{-2}\right\} \mathrm{d} \mu .
$$

The integrand of the functional $\mathcal{M}$ is singular at $\rho=0$. However, we have defined the space $\mathcal{B}$ only for functions $y$ with support in $\Omega_{\rho_{0}}$. Then, the domain of the integration of the terms in which $\partial y$ appears is in fact $\Omega_{\rho_{0}}$, and hence the integrand is clearly regular for those terms. Moreover, we have the following.

Lemma 2.1. Let $\varphi \in \mathcal{B}$, then the function $i_{\varphi}(t)$ is $C^{2}$ in the $t$ variable.

Proof. This is a straightforward computation. We will show the third derivative exists for all $t$. From equation (24), we compute

$$
i_{\varphi}^{\prime \prime \prime}(t)=\frac{1}{8 \pi} \int_{\mathbb{R}^{3}}\left(-2 \alpha^{3}|\partial Y|^{2}+6 \alpha^{2} \partial Y \partial y-3 \alpha|\partial y|^{2}\right) X^{-2} \mathrm{~d} \mu .
$$

The terms which contain $\partial y$ in (25) have support in $\Omega_{\rho_{0}}$, using inequalities (A.7) and (A.6) they can be easily bounded by the norm $\mathcal{B}$. The only term which does not contain $\partial y$ is given by

$$
-2 \alpha^{3}\left|\partial Y_{0}\right|^{2}
$$

To bound this term we use inequality (A.6).

In [6], we have proved that the extreme Kerr initial data are critical points of $\mathcal{M}$, that is we have

$$
i_{\varphi}^{\prime}(0)=0, \quad \text { for all } \varphi \in \mathcal{B} .
$$

In the following section, to prove that the second derivative is coercive, we will need the following auxiliary Hilbert space $\mathcal{H}$, which is defined in terms of the weighted Sobolev norms:

$$
\begin{aligned}
\|\alpha\|_{\mathcal{H}_{1}}^{2} & =\int_{\mathbb{R}^{3}}|\partial \alpha|^{2} \mathrm{~d} \mu+\int_{\mathbb{R}^{3}}|\alpha|^{2} r^{-2} \mathrm{~d} \mu, \\
\|y\|_{\mathcal{H}_{2}}^{2} & =\int_{\Omega_{\rho_{0}}}|\partial y|^{2} \rho^{-4} \mathrm{~d} \mu+\int_{\Omega_{\rho_{0}}}|y|^{2} \rho^{-6} \mathrm{~d} \mu,
\end{aligned}
$$


and its corresponding scalar products. Note that both the domain of integration and the weight functions are different in these definitions. The norm in $\mathcal{H}$ is defined by

$$
\|\varphi\|_{\mathcal{H}}^{2} \equiv\|\alpha\|_{\mathcal{H}_{1}}^{2}+\|y\|_{\mathcal{H}_{2}}^{2},
$$

with its corresponding scalar product. We have $\mathcal{B} \subset \mathcal{H}$.

The following weighted Poincare inequalities will be important in the proof of lemma 2.3.

Lemma 2.2. Let $\varphi \in \mathcal{H}$ and $\delta \neq 0$ a real number. Then

$$
\begin{aligned}
&|\delta|^{-1} \int_{\mathbb{R}^{3}}|\partial \alpha|^{2} r^{-2 \delta-1} \mathrm{~d} \mu \geqslant \int_{\mathbb{R}^{3}}|\alpha|^{2} r^{-2 \delta-3} \mathrm{~d} \mu, \\
&|\delta|^{-1} \int_{\Omega_{\rho_{0}}}|\partial y|^{2} \rho^{-2 \delta} \mathrm{d} \mu \geqslant \int_{\Omega_{\rho_{0}}}|y|^{2} \rho^{-2 \delta-2} \mathrm{~d} \mu .
\end{aligned}
$$

Proof. Inequality (31) is proved in [1] (theorem 1.3). Using a similar argument we will prove (32). It is sufficient to consider functions $y$ with compact support in $\Omega_{\rho_{0}}$. The key is the following identity:

$$
\Delta(\ln \rho)=0, \quad \text { in } \Omega_{\rho_{0}},
$$

where $\Delta$ is the Laplacian in $\mathbb{R}^{3}$. Testing this equation with $\rho^{-2 \delta} y^{2}$ gives

$$
\int_{\Omega_{\rho_{0}}} \partial\left(\rho^{-2 \delta} y^{2}\right) \partial(\ln \rho) \mathrm{d} \mu=0 .
$$

Which expands to

$$
\int_{\Omega_{\rho_{0}}} \rho^{-2 \delta-2} y^{2} \leqslant|\delta|^{-1} \int_{\Omega_{\rho_{0}}} \rho^{-2 \delta-1}|y|\left|\partial_{\rho} y\right| \mathrm{d} \mu,
$$

and Hölders inequality gives (32).

The following lemma shows that $i_{\varphi}^{\prime \prime}(t)$ is uniformly continuous with respect to the $\mathcal{H}$ norm.

Lemma 2.3. Let $0<t<1$. For every $\epsilon>0$ there exist $\eta(\epsilon)$ such that for $\|\varphi\|_{\mathcal{B}}<\eta(\epsilon)$, we have

$$
\left|i_{\varphi}^{\prime \prime}(t)-i_{\varphi}^{\prime \prime}(0)\right| \leqslant \epsilon\|\varphi\|_{\mathcal{H}}^{2}
$$

Proof. The proof is a straightforward but tedious calculation. Using (24) we calculate

$$
i_{\varphi}^{\prime \prime}(t)-i_{\varphi}^{\prime \prime}(0)=\int_{\mathbb{R}^{3}}(A+B+D) \mathrm{d} \mu
$$

where

$$
\begin{aligned}
& A=2 \alpha^{2}\left(\frac{|\partial Y|^{2}}{X^{2}}-\frac{\left|\partial Y_{0}\right|^{2}}{X_{0}^{2}}\right) \\
& B=-4 \alpha \partial y\left(\frac{\partial Y}{X^{2}}-\frac{\partial Y_{0}}{X_{0}^{2}}\right) \\
& D=|\partial y|^{2}\left(X^{-2}-X_{0}^{-2}\right)
\end{aligned}
$$


We decompose further $A$ and $B$ in the following way:

$$
A=A_{1}+A_{2}+A_{3}, \quad B=B_{1}+B_{2},
$$

where

$$
\begin{aligned}
& A_{1}=2 \alpha^{2} \frac{\left|\partial Y_{0}\right|^{2}}{X_{0}^{2}}\left(\mathrm{e}^{-2 t \alpha}-1\right), \\
& A_{2}=4 t \frac{\alpha^{2}}{X_{0}^{2}} \mathrm{e}^{-2 t \alpha} \partial Y_{0} \partial y, \\
& A_{3}=2 t^{2} \frac{\alpha^{2}}{X_{0}^{2}} \mathrm{e}^{-2 t \alpha}|\partial y|^{2}
\end{aligned}
$$

and

$$
\begin{aligned}
& B_{1}=-4\left(\mathrm{e}^{-2 t \alpha}-1\right) \alpha X_{0}^{-2} \partial y \partial Y \\
& B_{2}=-4 t \alpha X_{0}^{-2}|\partial y|^{2}
\end{aligned}
$$

In the following, we will bound each individual term. We will repeatedly use inequalities (A.6)-(A.12) given in appendix A for the extreme Kerr initial data.

Note that $A_{1}$ is the only term without $\partial y$, and hence the only term with support in $\mathbb{R}^{3}$, the other terms have support in $\Omega_{\rho_{0}}$. To bound $A_{1}$, we use inequality (A.6)

$$
\int_{\mathbb{R}^{3}} A_{1} \mathrm{~d} \mu \leqslant 2 C_{1}\left(\mathrm{e}^{2 \eta}-1\right) \int_{\mathbb{R}^{3}} \alpha^{2} X_{0}^{-2} \mathrm{~d} \mu \leqslant 2 C_{1}\left(\mathrm{e}^{2 \eta}-1\right)\|\varphi\|_{\mathcal{H}},
$$

where we have used

$$
|\alpha| \leqslant \sigma^{\beta}\|\alpha\|_{C_{\beta}^{1}\left(\mathbb{R}^{3}\right)} \leqslant\|\alpha\|_{C_{\beta}^{1}\left(\mathbb{R}^{3}\right)} \leqslant\|\varphi\|_{\mathcal{B}} \leqslant \eta .
$$

To bound the other terms, we will use inequality (A.7). For $A_{2}$, we have

$$
\begin{aligned}
\int_{\Omega_{\rho_{0}}} A_{2} \mathrm{~d} \mu & \leqslant 4 \mathrm{e}^{2 \eta} \eta \int_{\Omega_{\rho_{0}}} \alpha \frac{\partial Y}{X_{0}} \frac{\partial y}{X_{0}} \mathrm{~d} \mu \\
& \leqslant 4 \mathrm{e}^{2 \eta} \eta\left(\int_{\Omega_{\rho_{0}}} \alpha^{2} \frac{|\partial Y|^{2}}{X_{0}^{2}} \mathrm{~d} \mu\right)^{1 / 2}\left(\int_{\Omega_{\rho_{0}}} \frac{|\partial y|^{2}}{X_{0}^{2}} \mathrm{~d} \mu\right)^{1 / 2}, \\
& \leqslant 4 C_{1} \mathrm{e}^{2 \eta} \eta\|\varphi\|_{\mathcal{H}}^{2},
\end{aligned}
$$

where in the second line we have used Hölder inequality and in the third line inequalities (A.6) and (A.7).

The terms $A_{3}$ is simpler

$$
\int_{\Omega_{\rho_{0}}} A_{3} \mathrm{~d} \mu \leqslant 2 \mathrm{e}^{2 \eta} \eta^{2}\|\varphi\|_{\mathcal{H}}^{2}
$$

In the similar way, we get

$$
\begin{aligned}
\int_{\Omega_{\rho_{0}}} B_{1} \mathrm{~d} \mu & \leqslant 4\left(\mathrm{e}^{2 \eta}-1\right)\left(\int_{\Omega_{\rho_{0}}} \alpha^{2} \frac{|\partial Y|^{2}}{X_{0}^{2}} \mathrm{~d} \mu\right)^{1 / 2}\left(\int_{\Omega_{\rho_{0}}} \frac{|\partial y|^{2}}{X_{0}^{2}} \mathrm{~d} \mu\right)^{1 / 2} \\
& \leqslant\left(\mathrm{e}^{2 \eta}-1\right) C_{1}\|\varphi\|_{\mathcal{H}}^{2} .
\end{aligned}
$$


For $B_{2}$, we get

$$
\int_{\Omega_{\rho_{0}}} B_{2} \mathrm{~d} \mu \leqslant 4 \eta\|\varphi\|_{\mathcal{H}}^{2}
$$

Finally, for $D$ we have

$$
\int_{\Omega_{\rho_{0}}} D \mathrm{~d} \mu \leqslant\left(\mathrm{e}^{2 \eta}-1\right)\|\varphi\|_{\mathcal{H}}^{2}
$$

Since all the coefficients multiplying $\|\varphi\|_{\mathcal{H}}^{2}$ are smooth with respect to $\eta$ and go to zero as $\eta$ goes to zero we get (36).

\section{Positivity of the second variation}

From equation (24), it is far from obvious that the second variation evaluated at the critical point $u_{0}$ is positive definite. In order to prove that, the key ingredient is the following remarkable identity proved by Carter [3]. In terms of our variables, it has the following form:

$$
F+\alpha G_{v}^{\prime}+y G_{Y}^{\prime}+2 \alpha y G_{Y}-X^{-2} y^{2} G_{v}=\partial\left(\alpha \partial \alpha+y X^{-1} \partial\left(y X^{-1}\right)\right),
$$

where

$$
\begin{aligned}
& G_{v}(t)=\Delta v+X^{-2}|\partial Y|^{2}, \\
& G_{Y}(t)=\partial\left(X^{-2} \partial Y\right),
\end{aligned}
$$

the derivatives with respect to $t$ of these functions are given

$$
\begin{aligned}
& G_{v}^{\prime}(t)=\Delta \alpha+\left(2 \partial y \partial Y-2 \alpha \partial_{a} Y \partial^{a} Y\right) X^{-2}, \\
& G_{Y}^{\prime}(t)=\partial\left(X^{-2}(\partial y-2 \alpha \partial Y)\right),
\end{aligned}
$$

and the positive definite function $F$ is given by

$F(t)=\left(\partial \alpha+y X^{-2} \partial Y\right)^{2}+\left(\partial\left(y X^{-1}\right)-X^{-1} \alpha \partial Y\right)^{2}+\left(X^{-1} \alpha \partial Y-y X^{-2} \partial X\right)^{2}$

The identity (57) is valid for arbitrary $v, Y, \alpha, y$ and it is straightforward to check.

For $\varphi \in \mathcal{B}$, integrating by part we obtain

$$
-\int_{\mathbb{R}^{3}}\left(\alpha G_{v}^{\prime}+y G_{Y}^{\prime}\right) \mathrm{d} \mu=16 \pi i_{\varphi}^{\prime \prime}(t) .
$$

Equation (27) is equivalent to $G_{v}(0)=G_{Y}(0)=0$. If we integrate the identity (57) in $\mathbb{R}^{3}$ for $t=0$, the boundary terms on the right-hand side vanishes for all $\varphi \in \mathcal{B}$, then we get

$$
i_{\varphi}^{\prime \prime}(0)=\int_{\mathbb{R}^{3}} F(0) \mathrm{d} \mu \geqslant 0
$$

Moreover, $i_{\varphi}^{\prime \prime}(0)$ is strictly positive. Assume that we have $i_{\varphi}^{\prime \prime}(0)=0$ for some $\varphi \in \mathcal{B}$. Then, $F(0)=0$. We rewrite the second and the third terms in (62) in the following form:

$$
\left(X \partial \bar{y}+\bar{y} \partial X-X^{-1} \alpha \partial Y\right)^{2}+\left(X^{-1} \alpha \partial Y-\bar{y} \partial X\right)^{2} \geqslant 2^{-1} X^{2}(\partial \bar{y})^{2},
$$

where

$$
\bar{y}=\frac{y}{X^{2}} .
$$

From this, we deduce that $F(0)=0$ implies $\partial\left(y X_{0}^{-2}\right)=0$, and hence, by the assumption $\varphi \in \mathcal{B}$, we obtain $y=0$. Then we deduce $\alpha=0$ from the first term in (57). 
Equation (64) is a necessary condition for a local minimum, however it is not sufficient. The following lemma gives the sufficient condition used in the proof of theorem 1.1. Note that in order to formulate this coercive condition we need the auxiliary Hilbert space $\mathcal{H}$ defined in the previous section.

Lemma 3.1. There exists $\lambda>0$ such that for all $\varphi \in \mathcal{B}$ we have

$$
i_{\varphi}^{\prime \prime}(0) \geqslant \lambda\|\varphi\|_{\mathcal{H}}^{2}
$$

Proof. Let $\varphi \in \mathcal{B}$. Note that $i_{\varphi}^{\prime \prime}(0)$, as function of $\varphi$, defines a bilinear form $a(\varphi, \varphi)$ given by

$$
a(\varphi, \varphi) \equiv i_{\varphi}^{\prime \prime}(0)=\int_{\mathbb{R}^{3}} F(0) \mathrm{d} \mu,
$$

where $F$ is given explicitly by (62).

Inequality (67) is equivalent to the following variational problem:

$$
\lambda=\inf _{\varphi \in \mathcal{B}} \frac{a(\varphi, \varphi)}{\|\varphi\|_{\mathcal{H}}^{2}} .
$$

Since $a(\gamma \varphi, \gamma \varphi)=\gamma^{2} a(\varphi, \varphi)$ for every real number $\gamma$, this variational problem is equivalent to

$$
\lambda=\inf _{\varphi \in M} a(\varphi, \varphi)
$$

where

$$
M=\left\{\varphi \in \mathcal{B}:\|\varphi\|_{\mathcal{H}}^{2}=1\right\} .
$$

It is clear that $\lambda \geqslant 0$ because $a(\varphi, \varphi)$ is positive definite. We want to prove that $\lambda>0$.

Let us assume that $\lambda=0$. Then, there exists a sequence $\left\{\varphi_{n}\right\}$ such that

$$
\left\|\varphi_{n}\right\|_{\mathcal{H}}=1, \quad \text { for all } n
$$

and

$$
\lim _{n \rightarrow \infty} a\left(\varphi_{n}, \varphi_{n}\right)=0
$$

By inequality (65) we have

$$
\lim _{n \rightarrow \infty} a\left(\varphi_{n}, \varphi_{n}\right) \geqslant 2^{-1} \int_{\Omega_{\rho_{0}}} X_{0}^{2}\left(\partial \bar{y}_{n}\right)^{2}=0 .
$$

Using bounds (A.7), (A.9) and the Poincare inequality (32) we have

$$
\begin{aligned}
\int_{\Omega_{\rho_{0}}} X_{0}^{2}\left(\partial \bar{y}_{n}\right)^{2} \mathrm{~d} \mu & \geqslant \int_{\Omega_{\rho_{0}}} \rho^{4}\left(\partial \bar{y}_{n}\right)^{2} \mathrm{~d} \mu \\
& \geqslant 2 \int_{\Omega_{\rho_{0}}} \rho^{2}\left|\bar{y}_{n}\right|^{2} \mathrm{~d} \mu \\
& \geqslant \frac{2}{C_{2}^{2}} \int_{\Omega_{\rho_{0}}} \rho^{-6}\left|y_{n}\right|^{2} \mathrm{~d} \mu .
\end{aligned}
$$

Then,

$$
\lim _{n \rightarrow \infty} \int_{\Omega_{\rho_{0}}} \rho^{-6}\left|y_{n}\right|^{2} \mathrm{~d} \mu=0
$$


Replacing $\bar{y}_{n}$ by $X_{0}^{-2} y_{n}$ in (74) we get the inequality

$$
a\left(\varphi_{n}, \varphi_{n}\right)+\frac{1}{4} \int_{\Omega_{\rho_{0}}} X_{0}^{-2}\left|y_{n}\right|^{2}\left|\partial \ln X_{0}\right|^{2} \geqslant 4 \int_{\Omega_{\rho_{0}}} X_{0}^{-2}\left(\partial y_{n}\right)^{2} .
$$

From this inequality, using bounds (A.7), (A.9) and (A.12), we obtain

$$
a\left(\varphi_{n}, \varphi_{n}\right)+\frac{C_{3}}{4} \int_{\Omega_{\rho_{0}}} \rho^{-6}\left|y_{n}\right|^{2} \geqslant 4 C_{2} \int_{\Omega_{\rho_{0}}} \rho^{-4}\left(\partial y_{n}\right)^{2} .
$$

We apply (78) and (73) to get

$$
\lim _{n \rightarrow \infty} \int_{\Omega_{\rho_{0}}} \rho^{-4}\left|\partial y_{n}\right|^{2} \mathrm{~d} \mu=0
$$

and then we have

$$
\lim _{n \rightarrow \infty}\left\|y_{n}\right\|_{\mathcal{H}_{2}}=0 \text {. }
$$

In the previous inequalities, we have only used the second and the third terms of $F$, the first term implies

$$
\int_{\Omega_{\rho_{0}}} y_{n}^{2} X_{0}^{-4}\left|\partial Y_{0}\right|^{2} \mathrm{~d} \mu+a\left(\varphi_{n}, \varphi_{n}\right) \geqslant 2^{-1} \int_{\mathbb{R}^{3}}\left|\partial \alpha_{n}\right|^{2} .
$$

We have

$$
\int_{\Omega_{\rho_{0}}} y_{n}^{2} X_{0}^{-4}\left|\partial Y_{0}\right|^{2} \mathrm{~d} \mu \leqslant C_{1} \int_{\Omega_{\rho_{0}}} y_{n}^{2} r^{-2} \rho^{-4} \leqslant C_{1} \int_{\Omega_{\rho_{0}}} y_{n}^{2} \rho^{-6} \mathrm{~d} \mu,
$$

where in the last inequality we have used that $r \geqslant \rho$.

From (84), (83) and (78) we deduce

$$
\lim _{n \rightarrow \infty} \int_{\mathbb{R}^{3}}\left|\partial \alpha_{n}\right|^{2} \mathrm{~d} \mu=0
$$

and using the Poincare inequality (31), we finally obtain

$$
\lim _{n \rightarrow \infty}\left\|\alpha_{n}\right\|_{\mathcal{H}_{1}}=0 \text {. }
$$

Equations (86) and (82) contradict (72), hence we have that $\lambda>0$.

\section{Proof of theorem 1.1}

Proof. The proof follows standard arguments, see for example [9] chapter 5 and [11] chapter 40.

In lemma 2.1, we have proved that the function $i_{\varphi}(t)$ is $C^{2}$ with respect to $t$. The classical Taylor theorem for $t=1$ yields

$$
\mathcal{M}\left(u_{0}+\varphi\right)-\mathcal{M}\left(u_{0}\right)=i_{\varphi}(1)-i_{\varphi}(0)=\frac{i_{\varphi}^{\prime \prime}(\vartheta)}{2}, \quad 0<\vartheta<1 .
$$

To prove (6), we will show that $i_{\varphi}^{\prime \prime}(\vartheta) \geqslant 0$ and $i_{\varphi}^{\prime \prime}(\vartheta)=0 \Rightarrow \varphi=0$.

From lemma 2.3, we have that for every $\epsilon>0$ there exist $\eta(\epsilon)$ such that the following inequality holds:

$$
\left|i_{\varphi}^{\prime \prime}(\vartheta)-i_{\varphi}^{\prime \prime}(0)\right| \leqslant \epsilon\|\varphi\|_{\mathcal{H}}^{2},
$$

for every $\|\varphi\|_{\mathcal{B}}<\eta(\epsilon)$. From this inequality, we deduce

$$
i_{\varphi}^{\prime \prime}(0)-\epsilon\|\varphi\|_{\mathcal{H}}^{2} \leqslant i_{h}^{\prime \prime}(\vartheta)
$$

We use lemma 3.1 and inequality (89) to conclude that

$$
(\lambda-\epsilon)\|\varphi\|_{\mathcal{H}}^{2} \leqslant i_{\varphi}^{\prime \prime}(\vartheta) .
$$

Choosing $\eta(\epsilon)$ such that $\epsilon<\lambda$ the desired result follows. 


\section{Acknowledgments}

It is a pleasure to thank R Bartnik, R Beig, L Simon and R Schoen for valuable discussions. Special thanks to Todd Oliynyk for illuminating discussions concerning Carter identity. This work has been supported by the Sonderforschungsbereich SFB/TR7 of the Deutsche Forschungsgemeinschaft.

\section{Appendix. Extreme Kerr initial data}

Consider the extreme Kerr metric, with angular momentum $J$, in Boyer-Lindquist coordinates $(t, \tilde{r}, \theta, \phi)$. The corresponding potentials $X_{0}, Y_{0}$ are given by

$$
Y_{0}=2 J\left(\cos ^{3} \theta-3 \cos \theta\right)-\frac{2 J^{2} \cos \theta \sin ^{4} \theta}{\Sigma},
$$

and

$$
\begin{aligned}
X_{0} & =\left(\frac{\left(\tilde{r}^{2}+|J|\right)^{2}-r^{2}|J| \sin ^{2} \theta}{\Sigma}\right) \sin ^{2} \theta, \\
& =\left(\tilde{r}^{2}+|J|+\frac{2|J|^{3 / 2} \tilde{r} \sin ^{2} \theta}{\Sigma}\right) \sin ^{2} \theta,
\end{aligned}
$$

where

$$
\tilde{r}=r+\sqrt{|J|}, \quad \Sigma=\tilde{r}^{2}+|J| \cos ^{2} \theta,
$$

The function $v_{0}$ is given by the definition (23)

$$
v_{0}=\ln X_{0}-2 \ln \rho,
$$

where $\rho=r \sin \theta$.

From (A.1) and (A.2), we get the following inequality:

$$
\frac{\left|\partial Y_{0}\right|^{2}}{X_{0}^{2}} \leqslant C_{1} r^{-2} \text { in } \mathbb{R}^{3} \text {. }
$$

From equation (A.3), we obtain a lower bound for $X_{0}$

$$
\rho^{2} \leqslant X_{0} \quad \text { in } \mathbb{R}^{3} .
$$

To get an upper bound, we use equation (A.2) to obtain

$$
X_{0}^{2} \leqslant 16 \rho^{4}\left(1+\frac{\sqrt{|J|}}{r}\right)^{4} .
$$

From this equation we deduce

$$
X_{0}^{2} \leqslant C_{2} \rho^{4} \quad \text { in } \Omega_{\rho_{0}},
$$

where

$$
C_{2}=16 \rho^{4}\left(1+\frac{\sqrt{|J|}}{\rho_{0}}\right)^{4}
$$

Note that $\lim _{\rho_{0} \rightarrow 0} C_{2}=\infty$.

From equations (A.3) and (A.5), we obtain

$$
\left|\partial v_{0}\right|^{2} \leqslant C_{3}^{\prime} r^{-2} \text { in } \mathbb{R}^{3},
$$

where $C_{3}^{\prime}>0$ and then

$$
\left|\partial \ln X_{0}\right|^{2} \leqslant\left|\partial v_{0}\right|^{2}+4 \rho^{-2} \leqslant C_{3} \rho^{-2} \text { in } \mathbb{R}^{3},
$$

where $C_{3}=C_{3}^{\prime}+4$ and we have used that $r \geqslant \rho$. 


\section{References}

[1] Bartnik R 1986 The mass of an asymptotically flat manifold Commun. Pure Appl. Math. 39 661-93

[2] Brill D R and Deser S 1968 Variational methods and positive energy in general relativity Ann. Phys. 50 548-70

[3] Carter B 1971 Axisymmetric black hole has only two degrees of freedom Phys. Rev. Lett. 26 331-3

[4] Choquet-Bruhat Y and Marsden J E 1976 Solution of the local mass problem in general relativity Commun. Math. Phys. 51 283-96

[5] Dain S 2005 Angular momemtum-mass inequality for axisymmetric black holes Preprint gr-qc/0511101

[6] Dain S 2006 A variational principle for stationary, axisymmetric solutions of Einstein's equations Class. Quantum Grav. 23 6587-71 (Preprint gr-qc/0508061)

[7] Dain S, Lousto C O and Takahashi R 2002 New conformally flat initial data for spinning black holes Phys. Rev. D 65104038 (Preprint gr-qc/0201062)

[8] Friedman J L and Mayer S 1982 Vacuum handles carrying angular momentum; electrovac handles carrying net charge J. Math. Phys. 23 109-15

[9] Giaquinta M and Hildebrandt S 1996 Calculus of variations: I Grundlehren der Mathematischen Wissenschaften volume 310 (Fundamental Principles of Mathematical Sciences) The Lagrangian formalism (Berlin: Springer)

[10] Horowitz G T 1984 The positive energy theorem and its extensions Asymptotic Behavior of Mass and Spacetime Geometry (Corvallis, OR, 1983) (Lecture Notes in Physics vol 202) ed F J Flaherty (Berlin: Springer) pp 1-21

[11] Zeidler E 1985 Nonlinear Functional Analysis and its Applications: III Variational methods and optimization (Translated from the German by L F Boron) (New York: Springer) 